\title{
Constraining the nanohertz gravitational wave background with the Parkes Pulsar Timing Array
}

\section{Ryan Shannon}

CSIRO Astronomy and Space Science Australia email: sha355@csiro.au

\begin{abstract}
The direct detection of gravitational waves will usher in a new era of astrophysics, enabling the study of regions of the universe opaque to electromagnetic radiation or electromagnetically quiet. An ensemble of pulsars (referred to as a pulsar timing array) provides a set of clocks distributed across the Galaxy sensitive to gravitational waves with periods on the order of five years (frequencies of many nanohertz). Plausible source of gravitational waves in this frequency band include massive black hole binaries in the throes of mergers and oscillating cosmic strings. The stochastic gravitational wave background, the sum of gravitational waves emitted throughout the universe, is the most likely signal to be detected by a pulsar timing array.

While the detection of gravitational waves will be a milestone in pulsar astronomy, a constraining limit on the strength of the gravitational wave background can be used to constrain cosmological models and early Universe physics. Here we present a new algorithm that can be used to constrain the strength of the GWB with a pulsar timing array. We then apply this technique to Parkes Pulsar Timing Array observations and place a new limit on the strength of the GWB. We conclude by discussing the astrophysical implications of this limit and the prospects for detecting gravitational waves with pulsars.
\end{abstract}

